Reuter: Diese Stellen irisierten etwas. Vom Flugzeug aus konnte man sie gut sehen, weil die kleinen Windwellen dort nicht durchkamen.

Hellmann: Was hat die ganze Aktion ungefähr an Kosten verursadit?

REUTER: Wir haben für etwa 160000,- DM Emulgatoren gekauft. Ich darf übrigens den anwesenden Herren von der BP danken, daß sie uns 40000 Liter Emulgatoren kostenlos zur Verfügung gestellt haben. Ein Teil dieser Enulgatoren liegt noch in Cuxhaven für zukünttige Unglücksfälle greifbar. Die Kosten für die Schiffe miteingerechnet dürften eine Summe von etwa $600000,-D M$ ergeben haben. Darin ist aber der Verlust an Ol - ca. $15000 \mathrm{t}$ - nicht enthalten. Die restlichen $25000 \mathrm{t} \mathrm{O} 1$ mußten mit erheblichen Kosten aufgearbeitet werden. Ich habe mir berichten lassen, daß der Wert des Wracks und des Restöls größer war als die Kosten der Bergungsaktion; insofern hat sich audh finanziell die Bergung gelohnt.

\title{
Bildung von Emulsionen und der Einsatz von Mineralölemulgatoren bei Verölungen
}

Hellmann: Zur Einführung und Erklärung könnte man sagen: Milch ist eine Emulsion von Fett in Wasser. Man kann die Milch mit Wasser unbeschränkt verdünnen und kann so das Fett langsam zum „Verschwinden" bringen, wenn man nur genügend Wasser zugibt. Dieses Beispiel soll auch für die Olvernichtung herhalten. Man will durch Zugabe von gewissen Stoffen zum OI erreichen, daß es sich mit Wasser unbeschränkt vermischen läßt. Was die Natur bei der Milch vollbracht hat, ist technisch nicht so einfach. Jeder, der mit den Emulgatoren und Emulsionen zu tun gehabt hat, weiß, daß ganz bestimmte Bedingungen eingehalten werden müssen, daß es längst nicht genügt, einen bestimmten Stoff zum Ol zuzufügen und dann mit Wasser zu verdünnen. Die Firmen haben aber ihre Produkte unter dieser einfachen Voraussetzung lange Zeit angeboten. Wir haben bei allen untersuchten Produkten - es sind mehr als 20 gewesen - festgestellt, daß dieser Sachverhalt nicht so einfach ist, wie behauptet wurde. Die Emulgatoren sollen eine Vermischung des Oles mit dem Wasser erleichtern, d. h. sie müssen Eigenschaften des Wassers und des Oles besitzen. Die Emulgatoren setzen sich im wesentlichen aus einem organischen Lösungsmittel und einer aktiven Komponente, dem eigentlichen Emulgator, zusammen. Das Lösungsmittel hat verschiedene Funktionen zu erfüllen, die ich hier nicht näher erläutern möchte. Das Lösungsmittel madht aber volumenmäßig sehr viel des gesamten Produktes - bis zu $90 \%$ aus, so daß erklärlich ist, weshalb die Emulgatoren in starker Konzentration angewendet werden müssen. Unsere Versuche ergaben folgendes (vgl. Hellmann \& KNöpp 1967, HellmanN, KLEIN \& KNöpr 1966): Man kann eine Emulsion des Oles in Wasser nur erreichen, wenn man (1) einen Teil Ol auf einen Teil Emulgator gibt, (2) wenn die Kontaktzeit vor der Durchmischung genügend groß ist und (3) die Durchmischung O1/Emulgator/Wasser sehr intensiv ist. Dann kann man bei besonders guten Produkten eine Emulsion erhalten, die sich unbeschränkt mit Wasser vermischen läß $\mathrm{k}$, so daß man sagen kann, das Ol "verschwindet". Diese Versuche sind unter extrem günstigen Bedingungen in geschlossenen Systemen in Laboratorium durchgeführt worden, und zwar in einem Becherglas, in dem das OIl nicht ausweichen konnte, in dem aber das Ol immer wieder mit dem Emulgator reagieren mußte. Außerdem wurde bei 10000 bis 20000 Upm emulgiert. Wir haben versucht, die Ergebnisse auf die Natur zu übertragen und haben folgendes festgestellt: Es ist meistens nicht möglich, (1) eine ausreichende Menge Emulgator an das Ol heranzubringen, (2) die erforderliche Kontaktzeit Ol/Emulgator zu garantieren, weil das Wasser unter Umständen den Emulgator immer wieder herauswäscht und (3) eine ausreichende Turbulenz zu erzeugen, da das OOl bzw. die Ollache beim Mischen ausweicht. Auf die Schwierigkeiten, Riesenölfelder von allen Seiten zu besprühen und auf andere auftretende Schwierigkeiten möchte ich hier nicht zu sprechen kommen.

Gunkel: Sie sagten, Stabilität sei erst beim Verhältnis 1:1 erreicht. Gilt das für jedes Wasser? Sind die Verhältnisse im Süßwasser nicht günstiger?

Hellmann: Die Verhältnisse sind wechselnd und nicht voraussagbar; das Ergebnis hängt vom Emulgator ab. Es gibt Emulgatoren, die sich im Süßwasser günstiger und andere, die sich im Salzwasser günstiger auswirken (vgl. HellmanN \& KNöpp 1967). 
THON: Kann man die angewandten Emulgatoren in bestimmte Gruppen einordnen?

HellmanN: Ja, das könnte man, wenn die Hersteller uns darüber Auskunft gegeben hätten.

BLOKKER: Im Prinzip kann man zwei Typen von Emulgatoren unterscheiden: Bei dem einen Typ muß man nach dem Eingießen ins Wasser sehr stark rühren, bei dem anderen Typ, z. B. Schleifölen, braucht man nur schwach zu rühren. Die Aktivität dieser Emulgatoren ist so groß, daß eine starke Wirbelung an der OOl-Wasser-Oberfläche entsteht; man nennt das spontane Emulgierung. Für diese Emulgatoren braucht man ziemlich große Mengen. Wenn man von der Industrie Detergentien bezieht, sollte man den Zweck angeben. Wenn man darauf hinweist, daß spontane Emulgierung notwendig ist, dann ist die Chance, daß man nicht den richtigen Emulgator bekommt, schon kleiner.

SCHWEISFURTH: Um eine in der Technik verwendbare Ol-Wasser-Emulsion zu erhalten, wird nicht nur mit einem, sondern stets mit mehreren Emulgatoren gearbeitet. Ferner werden an das verwendete Wasser im Betrieb auch bestimmte Anforderungen gestellt, z. B. soll in manchen Fällen eine Enthärtung zur Erzielung einer stabilen Emulsion erforderlich sein. Bei der Herstellung eines sogenannten Konzentrates (O1 + Emulgatoren), wird rein empirisch vorgegangen - man gewinnt diesen Eindruck jedenfalls. Es kommt augenscheinlich darauf an, die einzelnen Emulgatoren in ihrer Art und Konzentration aufeinander abzustimmen. Bedenkt man dies, muß man sich wundern, $\mathrm{d} a ß$ im Meerwasser, auf dem Ol herumschwimmt, ein einziger Emulgator wirksam werden soll.

HeLlmann: Wir können in der Bundesanstalt keine Emulgatoren entwickeln; dazu haben wir auch gar nicht die technischen Möglichkeiten. Wir stellen lediglich fest, welche Mittel empfehlenswert bzw. abzulehnen sind.

SCHWEXSFURTH: Entscheidend ist wohl, daß die Ol-Emulgatoren-Konzentrate, wie sie in der metallverarbeitenden Industrie verwendet werden, bereits im Gemisch vorliegen und Wasser in dieses Gemisch hineingegeben wird. Bei einer auf dem Meer schwimmenden Olschicht müßte erst für eine innige Vermischung von $\mathrm{Ol}$ mit dem oder besser den Emulgatoren gesorgt werden!

Reuter: Wir spritzen die Mischung in einer Verdünnung von 1:10, oder wie die Hersteller vorschreiben, mit einem Feuerwehrschlauch bzw. ähnlichen Vorrichtungen hinein.

Schweisfurth: Gewiß, aber die Stärke des Strahles wird nicht ausreichen, um überall eine gleichmäßige Verteilung zu sichern.

REUTER: Nein, sicherlich nicht.

KüHL: Haben Sie denn bei Ihren 20 Proben diese Unterschiede gefunden, von denen eben gesprochen wurde?

Hellmann: Auf Wasseroberflächen habe ich keine spontane Emulsionsbildung gefunden. In einem ganz anderen Zusammenhang gelang mir dagegen eine spontane Emulgierung. In einem kleinen Aluminiumtopf wurde etwa $1 \mathrm{~g}$ Motorenöl auf dem Boden verteilt. Anschließend gaben wir eine Mischung von $2 \mathrm{~g}$ Moltoclar in Wasser auf den Olfilm. Auf Wasseroberflächen hatte sich keine Wirkung gezeigt (Verhältnis Moltoclar : Wasser $=1: 10$ ). Ich habe nicht erwartet, daß eine Emulsion entstehen würde. Die Mischung ließ ich einen halben Tag lang stehen, wobei sie vorher nod einmal mit Wasser verdünnt wurde. Ich bekam eine sehr gute Emulsion, wie ich sie bisher nie in Händen hatte.

BLOKKER: Wenn eine Emulsion entsteht, so zeigt es sich in der Praxis darin, daß sich beim Zugeben von Wasser eine milchähnliche Lösung bildet.

WALLHÄUSSER: Wir liefern nur die Emulgatoren. Andere Firmen probieren dann aus, welche Emulgatoren in welcher Mischung vorteilhaft sind, und sie liefern nachher dieses fertige Schleiföl-Emulgator-Gemisch zur Weiterverdünung an die metallverarbeitende Industrie.

BLoKker: Die meisten Emulsionen dieser Art sind sehr salzempfindlich. Wenn Sie kleine Mengen Salz hineinschütten, bricht die Emulsion zusammen.

WALLHÄUSSER: Ja, die Emulsionen sind empfindlich gegenüber Salz und Mikroorganismen. 
REUTER: Wenn diese milchige Wirkung ein Indiz ist für die Emulsionsbildung, so haben wir diese auch bei 10 facher Verdünnung feststellen können. Die milchige Wirkung entstand sofort, wenn der verdünnte oder unverdünnte Emulgator in die Olschicht gespritzt wurde.

\section{WALLHÄUSSER: War sie beständig?}

REUTER: Ob sie stabil blieb, konnten wir nicht mehr verfolgen.

Wallhäusser: Das ist aber doch das Wichtigste dabei! Was nützt dieser momentane Effekt, wenn die Emulsion nachher wieder bricht.

Hellmann: Die meisten Emulgatoren sind selbst in Wasser emulgierbar, sie liefern daher auch ohne Ol eine Trübung des Wasserkörpers. Man sollte von einer Ölemulgierung erst dann reden, wenn die Oltröpfchen einen Durchmesser von $20 \mu$ besitzen (vgl. Abb. D4).

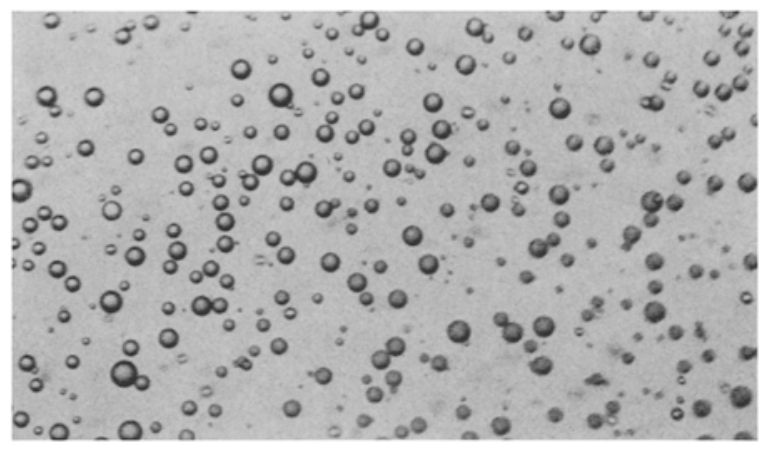

Abb. D4: Emulsion von Motorenöl HD SAE 20 in Wasser unter Verwendung von Emulgator SW. Tropfen kleiner als $10 \mu$

REUTER: Wir haben beobachtet, daß diese milchige Trübung sofort auftrat. Wurde sie dann besonders bei feinen Olfilmen - von den Schiffsschrauben durchwirbelt, so war bei verhältnismäßig ruhiger See zu erkennen, daß der Ólfilm in Tröpfchen von 1 bis $2 \mathrm{~mm}$ Größe auseinandergeplatzt war. Zwischen diesen Tröpfchen befand sich reines Wasser.

WallhäUsser: Aber das fließt wieder zusammen.

BLOKKER: Im Seewasser besteht die Schwierigkeit, daß des öfteren durch den Elektrolytgehalt die Emulsion wieder zerstört wird. Ich glaube, daß im Meerwasser nichtionogene Emulgatoren besser geeignet sind als ionogene.

Hellmann: Dies kann ich bestätigen. Zu der Beobachtung von Herrn Reuter möchte ich sagen, daß es sich um eine Olverteilung oder-zerteilung, also eine Vorstufe der Emulgierung, gehandelt haben muß.

REUTER: Ist die Olzerteilung nicht schon ein Effekt, den wir haben wollen? Wir beobachten bei der Olverdriftung zwei Erscheinungen, (1) die Windverdriftung, die auf die obersten Wasserschichten wirkt und (2) in der Nordsee die Tideströmung. Ist nicht schon eine Wirkung erzielt, wenn ein Teil des Ols, sei es durch Emulgierung oder Zerteilung, in größere Tiefen gelangt, wobei den Bakterien eine größere Angriffsfläche geboten wird?

WaLlHÄUSSER: Wenn diese Verteilung bestehen bleibt, dann ja.

REUTER: Wenn der Olfilm auseinandergerissen ist, kommt er in der Nordsee nicht mehr zusammen. Der eine Teil treibt unter Windeinfluß, zum Beispiel nach Nordosten, und der andere Teil kann mit der Tideströmung nach Westen treiben.

Wallhäusser: Wäre das nicht auch schon ohne Emulgatoren so der Fall? 
REUTER: Nein, dann würde das Ơl doch nur an der Oberfläche bleiben und nur dem Einfluß des Windes unterliegen.

Hellmann: $\mathrm{Zu}$ diesem Punkt sind noch eingehende Untersuchungen u. a. von Cuxhaven aus vorgesehen. Das Ergebnis ist abzuwarten.

Tomczak : Der Gezeiteneinfluß bedeutet nur, daß sich die Olfläche, die in Richtung des Windes treibt, verbreitert, aber es bedeutet nicht, daß die Teile auseinanderreißen. Der Teil, der durch die Tideströmung - in Ihrem Beispiel nach Westen geht - ist gleichzeitig der Driftströmung unterworfen; er geht also auch in Richtung des Windes mit. Und wenn die Tide wechselt, dann läuft der nach Westen gegangene Teil wieder nach Osten zurück. Der Gesamtweg ist also einfach nur das Resultat aus zwei Strömungen. Dies bedeutet eine Verbreiterung des Olbandes, nicht ein Auseinanderziehen. Die Winddrift ist an der Oberfläche am stärksten, aber sie reicht auch bis zu 2-3 m Tiefe. Sie läuft an der Oberfläche in Richtung des Windes und sollte nach der Theorie bis zu $45 \mathrm{Grad}$ von der Windrichtung abweichen. Diese Theorie gilt aber nicht für flache Meeresgebiete wie in der Deutschen Bucht. Deswegen haben Sie nur eine Verringerung der Winddrift mit größeren Tiefen.

Reuter: Nein, Herr Dr. Tomczak, da stimme ich nicht mit Ihnen überein! Meine Beobachtungen zeigten etwas anderes; wenn aus 2 bis $3 \mathrm{~m}$ Tiefe aus dem Lüfter eines Tankers Ol herauskam, dann zog es zunächst 500 bis $800 \mathrm{~m}$ in Richtung des Tidestromes. Sobald es an der Oberfläche war, geriet es in den Windeinfluß und wurde in Richtung des Windes unter 90 oder $170 \mathrm{Grad}$ verdriftet. Daß die Winddrift bis zu 3 Meter reichen soll, glaube ich nicht! 5 bis $20 \mathrm{~cm}$ erscheinen mir glaubwürdig.

ToMCZAK: Was ich sagte, das können Sie auch in Versuchen nachweisen! Ich will mich nicht zu sehr in Einzelheiten verlieren, da sie von unserem Thema "Emulgatoren " abweichen. Diese Daten sind experimentell nachgewiesen, und zwar (1) mit Oberflächendriftkarten für die oberen Millimeter der Meeresoberfläche, (2) mit Drifflaschen, die bis zu $20 \mathrm{~cm}$ Tiefe reichen, (3) mit Drachen, die in 2 bis $3 \mathrm{~m}$ Tiefe laufen, und (4) mit driftenden Schiffen, die bis zu $8 \mathrm{~m}$ Tiefe reichen. Die einzige Differenz, die Sie bei den verschiedenen Experimenten erhalten, ist der sogenannte Windfaktor. Er nimmt $a b$, je dicker die Schicht ist, die Sie im Auge haben. Und wenn Sie beobachtet haben, daß in einer bestimmten Tiefe das Öl anders läuft als an der Oberfläche, so ist das verständlich, denn die Winddrift in der Tiefe von 2 bis $3 \mathrm{~m}$ ist geringer (vielleicht $1,2 \%$ ) als an der Oberfläche (etwa $4 \%$ ) der Windgeschwindigkeit. Das heißt, was an der Oberfläche ist, läuft rasch in Richtung des Windes, was weiter tiefer ist, läuft langsamer, und die Ablenkung durch den Gezeitenstrom ist in dieser Tiefe stärker. Je dichter das OI an der Oberfläche ist, desto stärker wird die unmittelbare Einwirkung des Driftstromes.

Mann: Haben Sie, Herr Hellmann, Thre Versuche nur mit Süß wasser durchgeführt?

Hellmann: Nein, auch mit Salzwasser. Die Emulgatoren sprechen ganz verschieden auf Salzwasser an; der Salzgehalt hat nicht immer eine negative Wirkung, sondern oft eine positive. Man braucht beispielsweise weniger an Emulgatoren, wenn Meersalze vorhanden sind. Das ist ein interessanter Effekt (HellmanN 1967).

Schweisfurth: Wissen Sie, ob sie ionische oder nichtionische Emulgatoren hatten?

Hellmann: Darüber haben die Firmen keine genauen Angaben gemacht. Ich weiß aber, daß Emulgatoren, die ionisch aufgebaut sind, bevorzugt in Salzwasser brechen. Es gibt insgesamt drei Gruppen: die einen zerfallen im sauren, die anderen zerfallen im basischen Bereich; die dritte Gruppe ist unempfindlich gegenüber Ioneneinflüssen (vgl. HellmaNn, KleIN \& KNöpp 1966).

Brockis: Perhaps a few remarks on the use of detergents in Cornwall would be useful at this point. Experience has shown that where emulsifiers are used to desperse oil slicks at sea, efficient mixing is necessary for maximum effectiveness. It is simply not good enough just to tip quantities of emulsifier over the side of the ship and leave it. Churning up the oil - emulsifier - water mix with ships propellers has a very beneficial effect. There may be room for development of some sort of special despensing - mixing equipment or vessel for this purpose. 
When using detergents for cleaning beaches, it has been shown very clearly that it is most important to utilise the natural mixing effect of the sea. Detergent application across the beach parallel to the incoming tide should be timed so that the breaking wave action mixes the oil detergent - sand certainly within one hour of application, and the sooner the better.

GuNKEL: What is the stability of the emulsion formed under these conditions?

BROCKIS: I cannot give a specific answer to that. However, for practical purposes it is sufficient to say that application in this way is very effective. The mixing principle must also be applied when cleaning rocks. Where wave-breaking action will not cover soiled rocks, after applying detergents, it is necessary to direct a high pressure jet of water into the treated areas.

SchöвERL: What kind of emulsifiers were used in Cornwall?

Brockis: Several brands were used. As far as is known, most if not all of them were the non-ionic variety. It is believed that the majority were based on polyalkylene oxide-alkyl phenol condensates.

\section{Toxizität von Mineralölemulgatoren auf See- und Süßwasserorganismen}

KüHL: Die Mittel, die in Cuxhaven geprüft wurden, erhielt ich von Herrn Oberbaurat Reuter. Es waren dies: Oil-Ex, Anti-Oil, TS 5, Sillarit, Vecom B 24, PS 777, Moltoclar, J 502, Gamlen, Elimax, Slix, Peroclean. Als Testtiere dienten: die Larven von Spioniden, Balaniden und Schnecken; ferner Copepoden, Mysideen, Amphipoden, einige andere Plankter und schließlich Garnelen, Strandkrabben und Jungschollen (O-Gruppe). Die Versuche wurden im Cuxhavener Brackwasser (15 bzw. 20\% Salzgehalt) und Helgoländer Wasser (32\% Salzgehalt) durchgeführt. Die Letalitätsgrenzen lagen zwischen $2 \times 10^{-4}$ und $10^{-5}$ Verdünnung. Bei Mischungen mit Ol lagen die Grenzen in den gleichen Größenordnungen wie bei den reinen Emulgatoren. Die Versuche wurden beendet, wenn nach 6 Tagen keine sichtbare Schädigung mehr festzustellen war. Die Versuchstiere wurden dann in reines Seewasser zurückgesetzt. Bei den Bekämpfungen von Ölverschmutzungen durch Emulgatoren sind die Schadwirkungen im wesentlichen auf die Emulgatoren und in weitaus geringerem Maße auf das OI als solches zurückzuführen (vgl. KüHL \& MANN 1967).

MANN: Ole können nicht nur toxisch wirken, sondern haben auch noch andere unangenehme Eigenschaften, nämlich die Beeinflussung des Geschmackes von Fischen. Bekanntlich sind Elbfische vielfadh ungenießbar, wenn sie mit Olverschmutzungen in Berührung gekommen sind. Dies ist zum Beispiel vor Jahren bei Maifischen im Raum von Cuxhaven der Fall gewesen, auch von Plattfischen kennt man ähnliches. In diesem Zusammenhang sei darauf hingewiesen, daß auch Phenole den Fischen einen unangenehmen Beigeschmack beibringen können. Bekannt ist dies aus Gewässern, die durch phenolhaltige Abwässer verschmutzt wurden. Es ist daran zu denken, daß bei Vorhandensein von Emulgatoren im Wasser die Geschmacksbeeinflussung der Fische durch Ole und Phenole verstärkt werden kann, wie es von den Tensiden her bekannt ist. Hinzu kommt noch, daß nicht nur durch direkten Kontakt der Fische mit Ol und Olemulsionen der unangenehme Geschmack erzielt werden kann, sondern auch auf dem Wege über die Nährtiere. Werden zum Beispiel Forellen mit "schlecht" schmeckenden Fischen gefüttert, so nehmen die Forellen sehr schnell durch Speicherung der Geschmacksstoffe im Fett den "schlechten" Geschmack an. Auch die Eier von Geflügel, das mit beanstandeten Fischen gefüttert wurde, schmeckten nach Ölen und Phenolen (MaNN 1960). In Binnengewässern wurden schon neben Emulgatoren Bindemittel zur Bekämpfung von Olverschmutzungen mit Erfolg eingesetzt. Diese Mittel, bei denen es sich um Stoffe auf der Basis von Holzmehl, Torf, Kunststoff oder Gesteinsglas handelt, sind fischereilich unbedenklich (MANN 1966).

WallhäUsser: Haben Sie die Toxizität geprüft, wenn Sie beispielsweise an Kieselgur Ol absorbieren, und das sinkt ab? Geht das Ol von der Oberfläche des Kieselgurpartikels wieder ab?

ManN: Bei den Ölbindern muß man zwei Typen unterscheiden. Die eine Gruppe, die ich oben erwähnte, bleibt auch nach Aufnahme des OOls schwimmfähig und kann von der Oberfläche 